\title{
Recubrimientos superficiales con láseres de diodos de alta potencia
}

\author{
J.L. Molpeceres ${ }^{(*)}$, M.J. Tomás ${ }^{(*)}$, B. Pérez ${ }^{(*)}$ y L. Leal ${ }^{(*)}$
}

Resumen La posibilidad de disponer actualmente de láseres de diodos con una potencia de emisión óptica superior a $1 \mathrm{~kW}$ permite abordar un gran número de aplicaciones en el ámbito del procesado de materiales con láser, dominado tradicionalmente por los láseres de $\mathrm{CO}_{2}$ y Nd:YAG. En el presente trabajo, se muestra la aplicación de los láseres de diodos de alta potencia al recubrimiento de materiales metálicos con aleaciones de base cobalto y níquel. Se describen las características del sistema empleado y se muestran los resultados obtenidos.

Palabras clave: Láseres de diodos. Recubrimiento. Plaqueado. Tratamiento superficial con láser.

\section{Coating with high power diode lasers}

\begin{abstract}
Nowadays, high power diode laser systems are available in the market. These systems, based on arrays of small diode lasers, can achieve more than $1 \mathrm{~kW}$ of output power and are currently offering new possibilities in the field of laser material processing where $\mathrm{CO}_{2}$ and $\mathrm{Nd}$ :YAG lasers were used up to the present. The results obtained in some materials cladding based on cobalt and nickel alloys as additional materials is presented. The instrumental set-up used in these experiments are described.
\end{abstract}

Keywords: Diode lasers. Coating. Cladding. Laser surface treatment.

\section{INTRODUCCIÓN}

La evolución experimentada durante los últimos años por los diodos láser con potencias comprendidas en el rango de 20 a $50 \mathrm{~W}$, ha permitido incrementar su fiabilidad y prestaciones. Los últimos desarrollos en este campo han culminado con la salida al mercado de diodos láser que permiten construir matrices con una potencia de emisión óptica superior a $1 \mathrm{~kW}$ y con un reducido tamaño. Sirva como ejemplo el hecho de que las dimensiones de una matriz de $1.800 \mathrm{~W}$ pueden ser del orden de $100 \times 50 \times 50 \mathrm{~mm}(1)$.

El haz láser, que emerge con una alta divergencia de cada diodo, ha de ser colimado por un sistema óptico compuesto generalmente por microlentes cilíndricas que se integran en la matriz de diodos (2-5). Un conjunto de prismas y lentes dirigen y focalizan la energía liberada por cada diodo, superponiéndola en el plano focal, con el fin de incrementar la densidad de potencia liberada por el sistema. Como puede apreciarse en la figura 1, la distri-

(*) Centro de Tecnología Láser. Parque Tecnológico de Boecillo. 47151-Boecillo (Valladolid, España). (a)

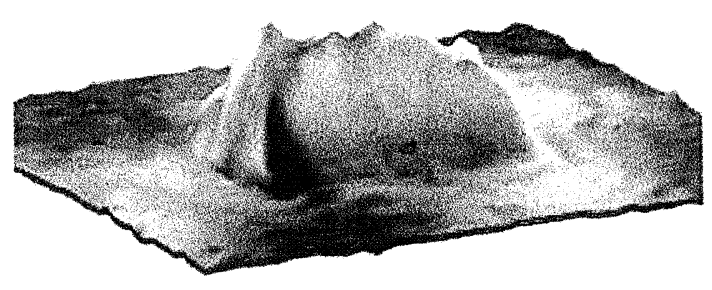

(b)

(c)
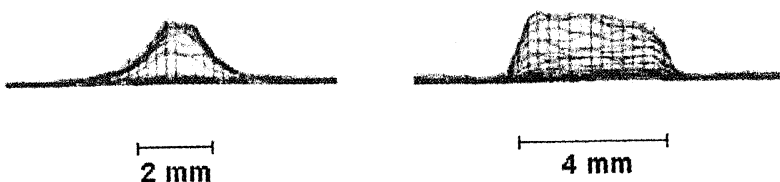

Fig. 1.- Distribución espacial de la energía liberada por un haz láser de alta potencia medida en el plano focal.

FIG. 1.- High power diode laser energy distribution in the focal plane.

bución espacial de energía resultante en dicho plano responde a una geometría rectangular. 
El tipo de procesos que un láser puede realizar en el campo del procesado de materiales depende en gran medida de la densidad de potencia que pueda suministrar y del tiempo de interacción con el material (6). Así, los diodos láser de alta potencia encuentran actualmente su mayor campo de aplicación en los tratamientos superficiales (temple, recubrimientos y aleaciones superficiales, mecanizado asistido por láser...) y en algunos procesos de soldadura de plásticos, componentes y terminales en circuitos electrónicos, etc. (7 y 8).

El sistema que se emplea en los trabajos que se describen a continuación puede focalizar hasta $1.200 \mathrm{~W}$, procedentes de una matriz de diodos, en un tamaño de $3,5 \times 2,4 \mathrm{~mm}$, con una longitud de onda de $940 \mathrm{~nm}$, y permite crear recubrimientos sobre materiales metálicos.

\section{PARTE EXPERIMENTAL}

Para el trabajo que se presenta, se eligió como material base una fundición gris laminar GG25

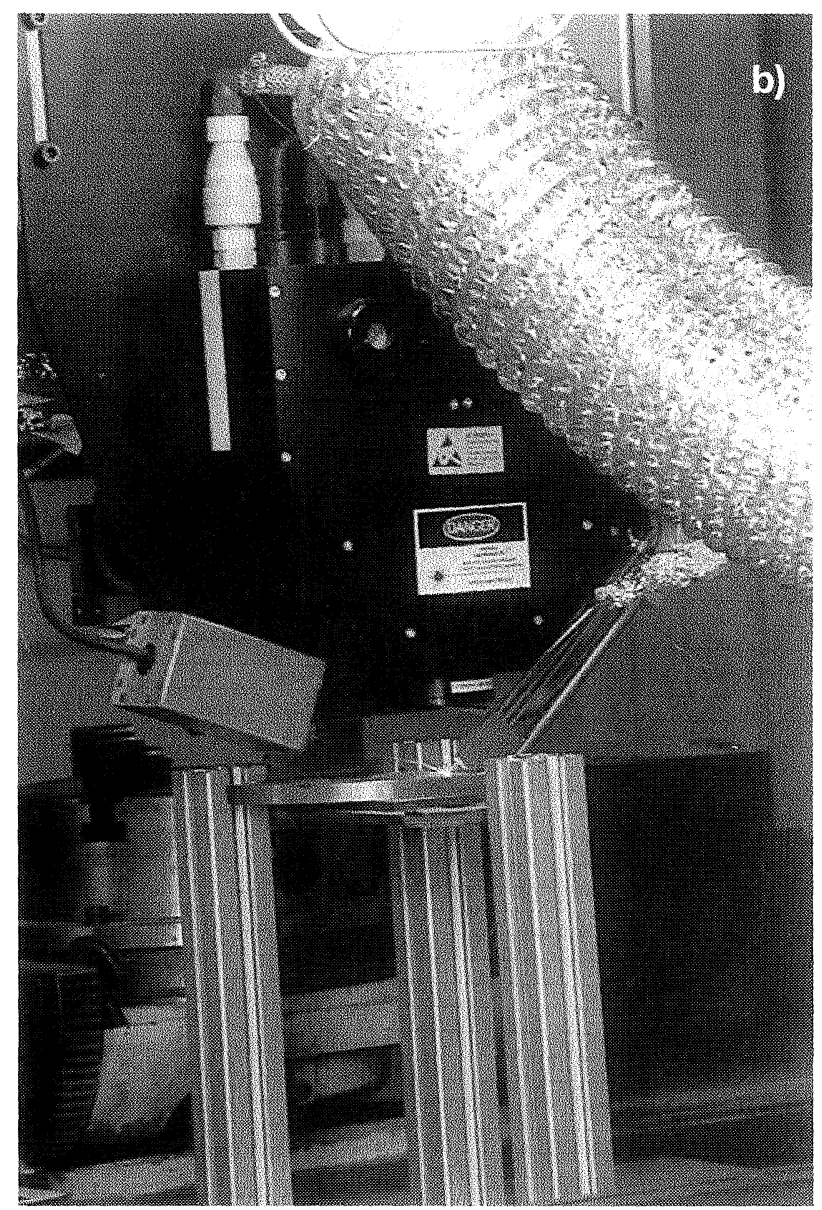

(fundición de hierro con $3,4 \%$ C, $2 \% \mathrm{Si}, 0,8 \%$ $\mathrm{Mn},<0,1 \% \mathrm{P}$ y $<0,15 \% \mathrm{~S}$ ) y como materiales de aporte dos aleaciones comerciales: Hoganäs 1620 (aleación de níquel con 1,5\% B, 0,05\% C, 0,8\% Fe y $2,6 \% \mathrm{Si}$ ) y Metco $18 \mathrm{C}$ (aleación de cobalto con $0,12 \% \mathrm{C}, 18,72 \% \mathrm{Cr}, 3,36 \% \mathrm{Si}, 27,7 \% \mathrm{Ni}$, $3,03 \% \mathrm{~B}, 5,81 \%$ Mo y $2,5 \% \mathrm{Fe})$.

El láser de diodos de alta potencia se instaló en una máquina herramienta de control numérico dotada de 4 grados de libertad ( $X Y Z+$ eje de giro) a la que se acoplaron una boquilla y un sistema comercial de suministro de material de aporte. Este dispositivo instrumental, utilizado para la realización del recubrimiento sobre la probeta de GG25, se muestra en la figura 2.

El proceso de recubrimiento se realizó en una sola etapa, inyectando el material en forma de polvo, con la asistencia de un flujo de gas, en el baño de fusión superficial generado por el láser (912). La superficie a tratar se limpió con chorro de arena antes de realizar el proceso de recubrimiento y no se realizaron tratamientos térmicos previos.
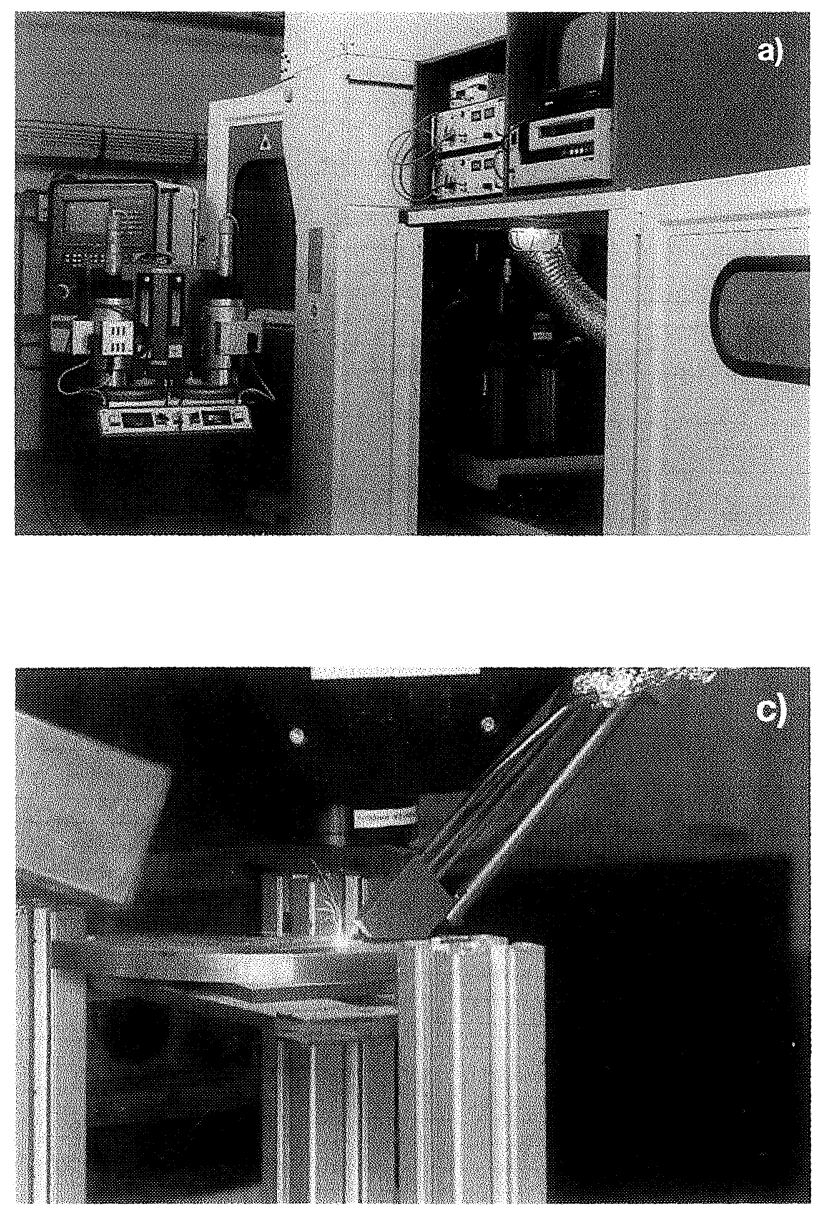

FIG. 2.- Dispositivo instrumental empleado para la realización de los experimentos de recubrimiento superficial con láser de diodos de alta potencia: $a$ ) vista general de la instalación. $b$ ) detalle del equipo láser de diodos. c) detalle del proceso de recubrimiento.

FIG. 2.- Experimental set-up for cladding with high power diode laser: a) General view of the facilities. b) detail of the diode laser equipment. c) detail of the cladding process. 
Los parámetros utilizados en el proceso se especifican en la tabla I.

\section{ANÁLISIS DE RESULTADOS}

Durante el proceso de enfriamiento de las piezas sometidas a pruebas de recubrimiento realizadas con Metco18C, se observó la aparición de grietas, probablemente generadas por una transformación martensítica en una zona del material base contigua al recubrimiento. Dicha transformación se traduce en el aumento de dureza observado en dicha zona durante los ensayos de microdureza a los que se sometieron las probetas correspondientes (Fig. 3). No se observó aparición de grietas en las pruebas de recubrimiento llevadas a cabo con aporte de Hoganäs 1620. La razón de este comportamiento puede encontrarse en la mayor ductilidad de este último material.

La aparición de poros en las capas de recubrimiento es un hecho directamente relacionado con varios factores. Por un lado, se apreció una gran influencia de la velocidad con que se lleva a cabo el proceso de recubrimiento. Por otro, se comprobó que la porosidad, probablemente debida a la existencia de láminas de grafito e impurezas en el material base, es menor cuanto menor es el grado de disolución del recubrimiento y cuanto mayor es el tiempo de interacción con la radiación debido a que, de esta forma, hay tiempo suficiente para que las burbujas generadas durante el proceso escapen del material fundido. Estos resultados son similares a los observados cuando se realizan recubrimientos superficiales con otros tipos de láser (por ej. láseres de $\mathrm{CO}_{2}$ ) en materiales base similares (13-18).

Para alcanzar distintos espesores de recubrimiento se variaron la velocidad del proceso y el flujo de material de aporte (Fig. 4). A una velocidad de proceso de unos $40 \mathrm{~mm} / \mathrm{min}$, se obtuvieron recu-

TABla I.- Parámetros empleados en los experimentos de recubrimiento realizados.

TABLE I.- Parameters used during the cladding experiments of this work.

\begin{tabular}{|c|c|}
\hline \multicolumn{2}{|c|}{ Parámetro experimental } \\
\hline potencia de láser, $\mathrm{W}$ & 1.000 \\
\hline diámetro de boquilla, $\mathrm{mm}$ & 2,5 \\
\hline flujo de gas de asistencia, $1 / \mathrm{min}(\mathrm{Ar})$ & 5 \\
\hline flujo de material de aporte, $\mathrm{g} / \mathrm{min}$ & $5-10$ \\
\hline velocidad de proceso $\mathrm{mm} / \mathrm{min}$ & $100-300$ \\
\hline
\end{tabular}

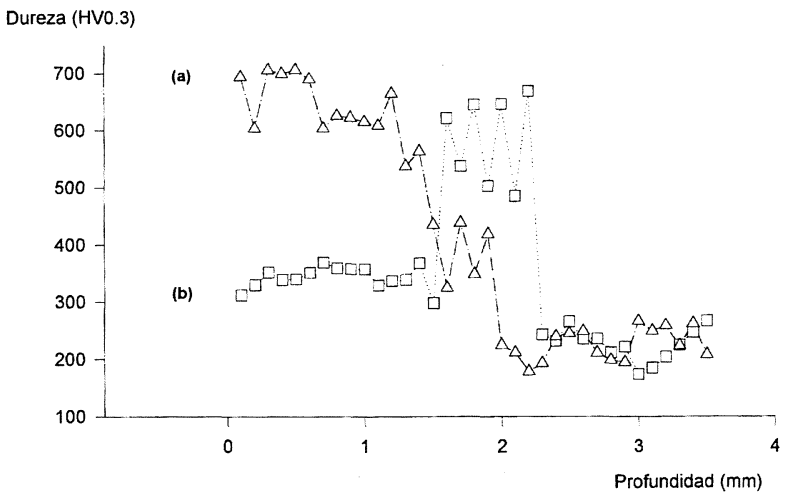

FIG. 3.- Variación de la dureza superficial con la profundidad alcanzada en una sección transversal del recubrimiento: a) recubrimiento de Metco 18C sobre GG25. $b$ ) recubrimiento de Hoganäs 1620 sobre GG25.

FIG. 3.- Microhardeness plot along a cross section of diode laser cladding on GG25 base material: a) additional material, Metco 18C. b) additional material: Hoganäs 1620.

brimientos libres de poros y grietas, con espesor de hasta $1 \mathrm{~mm}$ (Fig. 5).

\section{CONCLUSIONES}

En este trabajo se presenta un estudio preliminar en el que se muestran las posibilidades de aplicación de un nuevo tipo de láser de alta potencia a los procesos de recubrimiento superficial.

La utilización de los láseres de diodos de alta potencia en este tipo de procesos presenta como principal ventaja la de su fácil implementación en máquinas herramienta convencionales debido a su reducido tamaño. Su alto rendimiento energético y bajo coste de mantenimiento representan ventajas adicionales frente a los láseres de $\mathrm{CO}_{2}$ y Nd:YAG. $\mathrm{Su}$ menor longitud de onda y su geometría de distribución energética en el plano focal, hacen que este tipo de fuentes sean idóneas para las aplicaciones de recubrimiento superficial.

\section{Agradecimientos}

El trabajo que aquí se expone ha sido realizado con cargo a un proyecto CRAFT (BRST-CT965046), financiado por la Unión Europea. Qusiéramos manifestar nuestro agradecimiento a los socios colaboradores del citado proyecto y de forma especial a la empresa Lingotes Especiales, S.A. de Valladolid. 
Altura $(\mathrm{mm})$

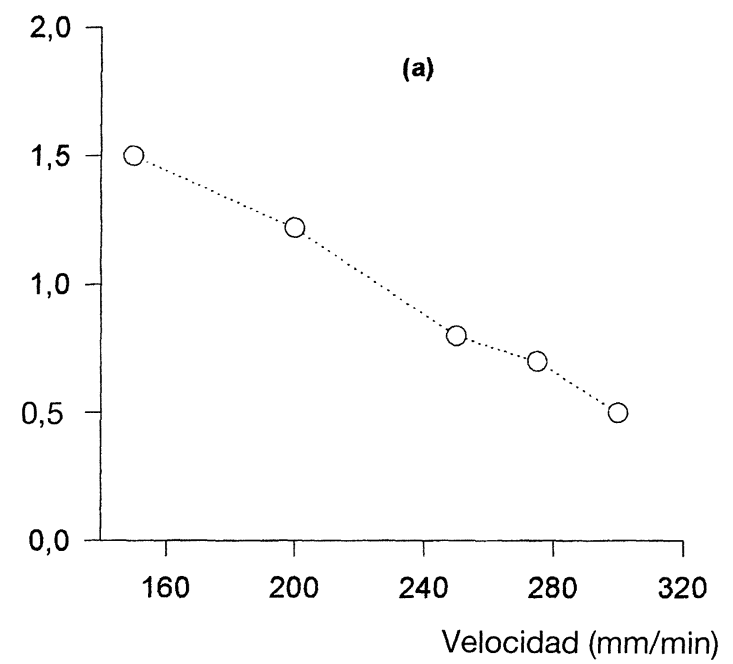

Altura (mm)

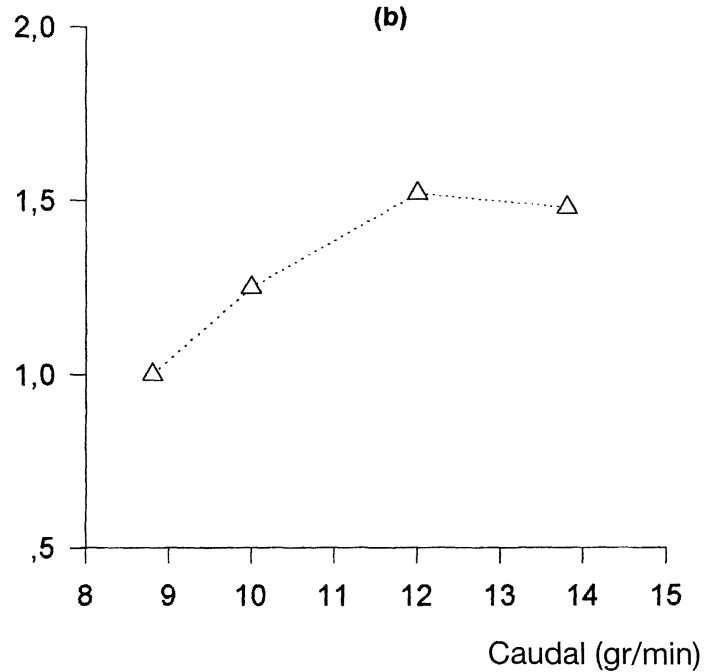

FIG. 4.- Variación de los espesores alcanzados en el recubrimiento de GG25 con Hoganäs 1620: $a$ ) en función de la velocidad del proceso (flujo de material de aporte $10 \mathrm{~g} / \mathrm{min}$ ). $b$ ) en función del flujo de material de aporte (velocidad del proceso $200 \mathrm{~mm} / \mathrm{min}$ ).

FIG. 4.- Coating thickness plot of diode laser cladding on GG25 with Hoganäs 1620:a) vs process speed (additional material flow: $10 \mathrm{~g} / \mathrm{min}$ ) b) vs additional material flow (process speed: $200 \mathrm{~mm} / \mathrm{min}$ )

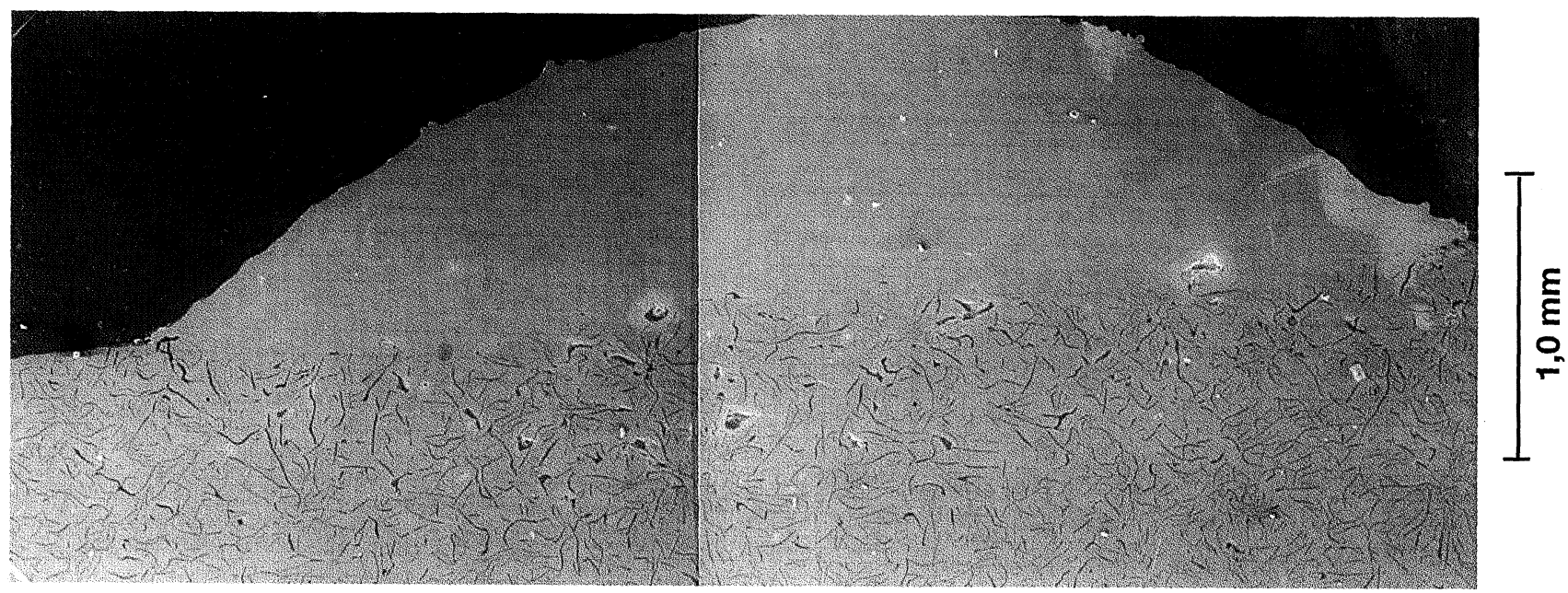

FIG. 5.- Sección transversal del recubrimiento obtenido sobre GG25 con una capa de Hoganäs 1620.

FIG. 5.-Cross section of a diode laser cladding of GG25 with Hoganäs 1620.

\section{REFERENCIAS}

(1) DILAS Diodenlaser GmbH. Catálogo de productos, 1997.

(2) KRAuSE, V. et al. Microchannel coolers for high power diodes in cooper technology. SPIE OE/LASE ‘94 Proc. 1994.

(3) MilsteR, T.D. Miniature optics for diode lasers and beam shaping, SPIE OE/LASE ‘94 Proc. 1994.

(4) BEACH, R.J. et al. Improved performance of high average power semiconductor arrays for applications in diode pumped solid state lasers, SPIE OE/LASE '95 Proc. 1995.

(5) ENDriz, J.G. et al. IEEE. J. Q. Electr. 28 (4), 1992: 952965.
(6) Steen, W.M. Laser material processing, Springer-Verlag. Londres. 1991: 172-219.

(7) Krause, V. et al. High power laser diodes as a beam source for materials processing, ECLAT 92' Proc. 1992.

(8) Loosen, P. Advanced concepts of using diode lasers in material processing, Proc. Laser and Material Processing, 3.097, 1997: 480.

(9) GASSER, A., Oberflächenbehandlung metallischer wekstoffe mit $\mathrm{CO}_{2}$-laser-strahlung in der flüssigen Phase, Conf. RWTH Aquisgrán (Alemania) 1993.

(10) Weerasinghe, V.M., Steen, W.M. Laser cladding by powder injection, Proc. Conf. Lasers in Manufacturing, IFS Publ. Ltd. (Ed. M. Kimmitt) 1983: 125-132. 
(11) Marsden, C.F., Frenk, A., Wagniere, J-D., Dekumbis, R. Effects of injection geometry on laser cladding. ECLAT '90 Proc. 1990.

(12) Ceri, W., Donati, W. y Fiorini, V. Laser cladding of cobalt-base powder on stainless and construction steels. Proc.SPIE. 650, 1986: 245-252.

(13) Megaw, J.H.P.C., Bransden, A.S., TrafFord, D.N.H., BELL, T. Surface Cladding by Multikilowatt Laser. Proc. 3rd Intern. Colloquium on Welding \& Melting by Electron \& Laser Beams, 1983.
(14) Tomlinson, W.J. y Bransden, A.S. Surf. Eng. 6 (4), 1990: 281-286.

(15) Gillner, A., Wissenbach, K., Kreutz, E.W. Laser Treatment of Materials, DGM Informationsgesellschaft Verlag. 1987: 205-212.

(16) Shen, L. y Chenglao, L. Wear. 147, 1991: 195-206.

(17) Steen, W.M. y Zhen da Chen, West D.R.F. The Industrial Laser Annual Handbook, Penn Well Publ. Co. 1987: 80-96.

(18) Zàrubova, N., Kraus, V. y Cermak, J. J. Mater.Sci. 27, 1992. 